\title{
Risco potencial do uso de medicamentos durante a gravidez e a lactação
}

\author{
Potential risk of medication during pregnancy and lactation
}

\author{
Alinne Souza RIBEIRO, Melquesedec Vieira SILVA, \\ Priscilla Gomes GUERRA, Ketene Werneck SAICK, \\ Michele Pereira ULIANA, Ricardo LOSS* \\ Faculdade Presidente Antônio Carlos de Aimorés - FUPAC/UNIPAC, \\ Minas Gerais, Brasil. E-mail: ricardoloss@ymail.com
}

\section{ABSTRACT}

The use of medication during pregnancy and lactation has always represented a challenge to health professionals, since it involves potentially harmful action not only for women but also for the fetus. Most drugs given to pregnant women crosses the placenta and expose the developing embryo to its pharmacological effects in infants and may occur at its transfer into breast milk. The aim of this study was to analyze the potential risks to women caused by the use of drugs commonly dispensed in the city of Aimorés (MG), if they were to be administered in pregnancy and breastfeeding period. We evaluated 21 selected drugs in the city of Aimorés, based on the most dispensed among women, identified by pharmacists of 10 municipal pharmacies. Drugs were classified according to the risk category of the FDA (Food and Drug Administration). Of the drugs studied, 4.76\% fell within the category of risk The FDA, in category B, $23.8 \%$. In C, $47.61 \%$ may have teratogenic effects or toxic to embryos, while in D $19.04 \%$ were identified drugs that highlights risk to human fetuses. In class X, only $4.76 \%$ of the drugs were identified. It was observed that most drugs represents a considerable risk to the fetus and the mother infant case use these drugs during pregnancy and lactation. It was found that the use should be better monitored by prescribers and the presence of pharmaceutical intervention in the dispensation is of paramount importance

KEYWORDS: Pregnancy; Lactation; Drugs

\section{RESUMO}

O uso de medicamentos na gestação e lactação sempre representou um desafio para os profissionais de saúde, uma vez que implica ação potencialmente danosa não apenas para a mulher, mas também para o concepto. A maioria dos fármacos administrados às mulheres grávidas atravessa a barreira placentária e expõe o embrião em desenvolvimento a seus efeitos farmacológicos, nas lactentes, podendo ocorrer a sua transferência para o leite materno. O objetivo do trabalho foi de analisar os riscos potenciais às mulheres, causados pela utilização dos fármacos comumente dispensados na cidade de Aimorés (MG), caso estes viessem a ser administrados em período de gestação e amamentação. Foram avaliados 21 medicamentos selecionados na cidade de Aimorés, tendo como base os mais dispensados entre as mulheres, identificados por meio de farmacêuticos de 10 farmácias municipais. Os medicamentos foram classificados de acordo com a categoria de risco da FDA (Food and Drug Administration). Dos fármacos estudados, 4,76\% se enquadrou na categoria de risco A da FDA, na categoria B, 23,8\%. Na C, 47,61\% podem apresentar efeitos teratogênicos ou tóxicos para os embriões, enquanto na $\mathrm{D}$ foram identificados $19,04 \%$ fármacos que evidencia risco para os fetos humanos. Na categoria $\mathrm{X}$, apenas $4,76 \%$ dos fármacos foram identificados. Observou-se que a maioria dos fármacos representa um risco considerável para o feto e o lactente caso a mãe utilize esses medicamentos durante o período de gestação e lactação. Constatou-se que o uso deve ser melhor monitorado pelos prescritores e a presença da intervenção farmacêutica na dispensação é de suma importância.

PALAVRAS-CHAVES: Gravidez; Lactação; Fármacos 


\section{INTRODUÇÃO}

A maior parte das gestantes e lactantes não está adequadamente informada sobre os riscos da utilização de medicamentos durante o período de gestação e aleitamento. A carência de informações e a complexidade dos diversos fatores que determinam a escolha de um medicamento para uso durante a gestação e lactação reforçam a preocupação sobre a automedicação nesse período e justificam a realização de um estudo que procure estabelecer um perfil dessa prática pelas nutrizes (1).

Durante o período de gestação e amamentação, alguns princípios básicos devem ser observados pelos profissionais de saúde para a correta prescrição de medicamentos, tais como relação dos riscos e benefícios, experiência prévia com o fármaco e suas propriedades (como meia-vida), dose recomendada, via e horário de administração, tempo de ação e níveis séricos, entre outros (2).

A Organização Mundial de Saúde (OMS) afirma que a automedicação ocupa um importante lugar no sistema de cuidados da saúde. Contudo, vários estudos consideram a automedicação motivo de grande preocupação, especialmente entre mulheres brasileiras na faixa etária entre 16 e 45 anos, nutrizes em potencial. Estudos têm demonstrado que os medicamentos mais comumente usados em automedicação na gestação incluem os analgésicos $(42,1 \%)$, antiácidos $(7,0 \%)$ e medicamentos para resfriados ou alergias $(6,0 \%)$. Outros estudos realizados no Brasil apontam uma estimativa de que $83,8 \%$ e $97,6 \%$ das gestantes estão expostas a, pelo menos, um medicamento durante a gestação $(3,4)$.
Desde a tragédia da talidomida, por volta das décadas de 50 e 60, a comunidade científica tem se focado na questão da segurança no uso de novos fármacos durante a gestação, pois, de fato, pouco se sabe sobre o efeito dos medicamentos no feto humano. Essa situação decorre da impossibilidade de se realizarem testes em humanos e da dificuldade de extrapolar os resultados obtidos em ensaios pré-clínicos in vitro e in vivo. Desse modo, cada vez é maior a importância e necessidade de estudos clínicos mais rigorosos antes da liberação de medicamentos para o consumo e elaboração de estudos que orientem os médicos no momento da prescrição (4).

O primeiro trimestre de gestação é o mais delicado e o de maior risco de ação danosa para o feto, por que é nessa fase que ocorrem as principais transformações embriológicas, sendo, portanto, imprescindível ser redobrado o cuidado na administração de medicamentos durante o período embriogênico $(5,6)$.

Quando se utiliza um fármaco durante o período da gestação, deve-se avaliar sempre o fator risco-benefício para mãe e feto. O medicamento de escolha deve ser aquele que não possui efeito teratogênico ou qualquer alteração funcional. Com o objetivo de orientar e auxiliar o prescritor na escolha terapêutica mais adequada para a gestante, desde 1975 a agência americana FDA (Food and Drug Administration) adota a classificação de medicamentos conforme o risco associado ao seu uso durante a gravidez, que são classificados em 5 categorias (A, B, C, D e X), crescentemente, de acordo com o grau de riscos à gestação, tomando por base, predominantemente, o primeiro trimestre de gravidez, como podemos observar na Tabela $1(4,7,8,9)$.

Tabela 1: Classificação de Risco dos Medicamentos para Uso na Gravidez de acordo com a FDA (Food and Drug Administration)

\begin{tabular}{|l|l|}
\hline Categoria de risco A & Estudos em mulheres não demonstraram risco para o feto no primeiro e demais trimestres; \\
\hline Categoria de risco B & Estudos em animais não demonstraram risco fetal, mas não há estudos no ser humano; \\
\hline Categoria de risco C & $\begin{array}{l}\text { Relatos em animais revelaram efeitos adversos no feto. Não há estudos controlados em } \\
\text { mulheres e animais. Os fármacos devem ser ministradas somente se o benefício justificar } \\
\text { o potencial teratogênico; }\end{array}$ \\
\hline Categoria de risco D & $\begin{array}{l}\text { Há evidência positiva de risco fetal e humano, porém os benefícios do uso em gestantes } \\
\text { podem ser aceitáveis; }\end{array}$ \\
\hline Categoria de risco X & $\begin{array}{l}\text { Estudos em animais ou seres humanos revelaram efeitos deletérios sobre o feto que ultra- } \\
\text { passam os benefícios. }\end{array}$
\end{tabular}

Fonte: Briggs (2002) 
Embora o conhecimento a respeito de fármacos na lactação tenha sido muito ampliado, ainda não se conhecem os efeitos colaterais para as crianças amamentadas de muitos fármacos utilizadas pela nutriz. Além disso, os efeitos de muitos fármacos novos ainda não foram devidamente estudados ou apresentam divergências na literatura quando utilizados na lactação. Portanto, surge a necessidade de atualizações constantes sobre o uso de medicamentos durante a amamentação, visando racionalizar esse uso e proteger o aleitamento materno (10).

Dessa forma, este trabalho visa à análise do risco do uso dos medicamentos comumente utilizados por mulheres na cidade de Aimorés (MG), caso estes fossem utilizados durante o período de gravidez e lactação.

\section{MATERIAIS E MÉTODOS}

Inicialmente foi feito um levantamento dos fármacos mais dispensados na cidade de Aimorés - Minas Gerais, de acordo com informações dos farmacêuticos de 10 farmácias municipais. Os fármacos selecionados foram estudados quanto ao seu potencial risco em gestantes e lactentes de acordo com a classificação de risco da FDA nas classes: A, B, C, D e X e nas classes farmacológicas respectivas.

\section{RESULTADOS}

A Tabela 2 apresenta os 21 fármacos selecionados, a correlação entre as classes farmacológicas e suas respectivas indicações no uso na gravidez e lactação de acordo com a FDA.

Dos fármacos selecionados, $1(4,76 \%)$ se enquadrou na categoria de risco A, considerada a mais segura. Na categoria B, $5(23,8 \%)$ fármacos se enquadram nos que ainda estão em estudo em humanos sobre seus efeitos adversos. Na C, 10 (47,61\%) estão no grupo que pode apresentar efeitos teratogênicos ou tóxicos para os embriões, enquanto na $\mathrm{D}$ foram identificados $4(19,04 \%)$ fármacos que estão na categoria que evidencia risco para os fetos humanos, mas os benefícios em certas situações, como por exemplo, nas doenças graves ou que põem em risco a vida e para as quais não existe outra alternativa terapêutica, podem fazer com que o uso durante a gravidez esteja justificado, apesar dos riscos. Na categoria X, apenas $1(4,76 \%)$ fármaco foi identificado, representando alterações fetais ou evidência de aumento no risco para o feto com base na experiência em humanos.

\section{DISCUSSÃO}

A ingestão de medicamentos nocivos ao feto se deve pelo fato de que a maioria dos mesmos tem a capacidade de atravessar a placenta, atingindo a circulação fetal. O uso de medicamentos durante a gravidez compreende uma situação única, na qual estão envolvidos dois organismos: a mãe e o feto. No feto, podem ocorrer transformações reversíveis e irreversíveis, como a alteração genética, a qual pode resultar a teratogênese (11).

Embora a etiologia das malformações congênitas seja em grande parte desconhecida, estima-se que de 2 a $5 \%$ são associadas aos medicamentos e, portanto, potencialmente evitáveis (12).

Ao prescrever um medicamento a ser utilizado durante o período de gestação e lactação, o prescritor deve informar ao seu paciente a classificação de risco e os transtornos causados pelos mesmos, levando em conta que estudos em animais não podem ser totalmente generalizados para a espécie humana (13).

Diversos fatores sócio-demográficos têm sido associados com o uso de medicamentos durante a gestação. Populações de países em desenvolvimento, como o Brasil, apresentam características sociais e econômicas muito peculiares para a compreensão de potenciais riscos teratogênicos aos quais uma gestante possa estar exposta. Essas características incluem níveis educacionais e econômicos baixos da população, alta incidência de doenças infectocontagiosas e carenciais, a facilidade em se obter medicamentos que exigem prescrição médica e, principalmente, a prática frequente e sem controle de automedicação (4).

Os Antibióticos Azitromicina, Clindamicina e Eritromicina, enquadraram-se na categoria $\mathrm{B}$, sendo contra-indicado seu uso na gravidez e lactação. Portanto, o fármaco somente deverá ser utilizado nos pacientes quando alternativas adequadas não estiverem disponíveis(14).

Os anti-inflamatórios Ácido Acetilsalicílico (AAS) e Nimesulida se encaixaram na categoria de risco C. A atividade antiplaquetária do AAS apresenta um risco potencial e já foram publicados relatos de doenças hemorrágicas em neonatos cujas mães fizeram uso de AAS durante a gestação. Recomenda-se a consulta com o médico antes da administração do AAS durante o período gestacional. O uso da Nimesulida está associado a uma incidência maior de hipertensão pulmonar, oligúria, oligoâmnio, aumento do risco de sangramento, atonia uterina e edema periférico. Casos isolados de insuficiência renal em recém-nascidos de mães tratadas com Nimesulida durante a gravidez têm sido relatados. Portanto, não deve ser administrada durante a gravidez ou em mulheres que estejam amamentando (15). 
Tabela 2: Classes farmacológicas dos medicamentos e suas respectivas indicações de uso na gravidez e lactação.

\begin{tabular}{|c|c|c|}
\hline Classe farmacológica & Medicamentos & Indicação e Classificação de acordo com a FDA \\
\hline Antibióticos & $\begin{array}{l}\text { Azitromicina, } \\
\text { Eritromicina, } \\
\text { Clindamicina; }\end{array}$ & $\begin{array}{l}\text { Categoria de risco B, sendo utilizados somente com orienta- } \\
\text { ção médica; }\end{array}$ \\
\hline Antiinflamatórios & $\begin{array}{l}\text { Ácido Acetilsalicílico, } \\
\text { Nimesulida; }\end{array}$ & $\begin{array}{l}\text { Categoria de risco } C \text {, apresentando um grau elevado em tera- } \\
\text { togênicas no feto; }\end{array}$ \\
\hline Anti-hipertensivos & $\begin{array}{l}\text { Captopril, } \\
\text { Metildopa, } \\
\text { Nifedipino, } \\
\text { Atenolol; }\end{array}$ & $\begin{array}{l}\text { Apresentaram categoria C e D, com grau de toxicidade elevado } \\
\text { e outros não podendo descartar a possibilidade de danos para } \\
\text { o feto; }\end{array}$ \\
\hline Antianêmicos & Ácido Fólico; & Categoria A, o recomendado seu uso durante a gravidez. \\
\hline Anticoagulantes & Warfarina; & Categoria X, ocasionando má formações de crianças. \\
\hline Antipsicóticos & Haloperidol; & $\begin{array}{l}\text { Categoria } C \text {, nenhum aumento significante nas anormalidades } \\
\text { fetais foi associado com o uso; }\end{array}$ \\
\hline Diuréticos & $\begin{array}{l}\text { Hidroclorotiazida, } \\
\text { Furosemida, } \\
\text { Espironolactona; }\end{array}$ & $\begin{array}{l}\text { Categorias B, C e D respectivamente, com riscos e danos que } \\
\text { podem superar os benefícios, como a inibição do parto ou da } \\
\text { lactação; }\end{array}$ \\
\hline Corticóides & Prednisona; & $\begin{array}{l}\text { Categoria } B \text {, podendo atravessar a barreira placentária e tam- } \\
\text { bém passar para o leite materno; }\end{array}$ \\
\hline Antigases & Simeticona; & $\begin{array}{l}\text { Categoria } C \text {, contudo não se têm informações suficientes na } \\
\text { literatura que comprovem algum efeito teratogênico; }\end{array}$ \\
\hline Antifúngicos & Cetoconazol; & $\begin{array}{l}\text { Atravessa a placenta e pode provocar problemas fetais du- } \\
\text { rante o primeiro trimestre da gravidez. Recomenda-se não } \\
\text { prescrevê-lo durante a gravidez e a lactação. }\end{array}$ \\
\hline Antiparasitários & Mebendazol; & $\begin{array}{l}\text { Categoria C. Possíveis riscos associados à prescrição de Me- } \\
\text { bendazol durante a gravidez devem ser pesados contra os be- } \\
\text { nefícios terapêuticos esperados. }\end{array}$ \\
\hline Retinóicos & Isotretinoina; & $\begin{array}{l}\text { Categoria } D \text {, causando a teratogenia, e geração de defeitos } \\
\text { permanentes no feto; }\end{array}$ \\
\hline Antidepressivos & Amitriptilina; & $\begin{array}{l}\text { Categoria } C \text {, os possíveis benefícios devem ser confrontados } \\
\text { contra os eventuais riscos para a mãe e a criança. }\end{array}$ \\
\hline
\end{tabular}

Fonte: FDA: Food And Drug Administration. (2010)

Os anti-hipertensivos Captopril, Metildopa, Nifedipino e Atenolol, apresentaram categorias C e D. Quando usados na gravidez durante o segundo e terceiro trimestres, os inibidores da enzima conversora de angiotensina (ECA) podem causar danos ao desenvolvimento e mesmo morte fetal. Na análise da Metildopa, não houve evidência clínica de que causasse anormalidades fetais ou afetasse o recém-nascido. Não se recomenda o uso do Nifedipino durante a gestação e lactação. O Atenolol atravessa a barreira placentária e aparece no sangue do cordão umbilical, e ocasiona acúmulo significativo no leite materno $(16,17)$.

O Ácido Fólico entra na classe dos antianêmicos, enquadrado na categoria de risco A, desempenhando importante função durante a gestação, pois ajuda a formar as células brancas e vermelhas do san- gue, e não apresenta contraindicações no período de lactação (18).

$\mathrm{Na}$ categoria de risco X, encontramos a Warfarina, um anticoagulante, sendo expressamente proibido seu uso durante a gravidez e lactação. Portanto, é contraindicado em gestantes ou mulheres em idade fértil, uma vez que o fármaco atravessa a barreira placentária podendo causar hemorragia fatal ao feto. Ainda houve relatos de más formações de crianças nascidas de mães que foram tratadas com Warfarina durante a gravidez (19).

O antipsicótico Haloperidol apresentou categoria de risco $\mathrm{C}$, mas nenhum aumento significante nas anormalidades fetais foi associado com o uso, porém, é excretado no leite $(20,21)$.

Em relação à classe dos diuréticos, a Hidroclo- 
rotiazida com risco $\mathrm{B}$, atravessa a placenta, e as concentrações atingidas na veia umbilical se aproximam às do plasma materno; é excretada no leite materno, o que pode impedir a lactação. A Furosemida apresentou risco $\mathrm{C}$, e não há estudos que comprovem o seu efeito durante a gravidez, mas deve ser evitado o uso. Tal medicamento passa para o leite materno, inibindo a lactação. A Espironolactona pertence à categoria $\mathrm{D}$, podendo atravessar a barreira placentária, diminuir a perfusão placentária, aumentar a inércia uterina e inibir o trabalho de parto; seu uso deve ser evitado durante a lactação, sendo estabelecido um método alternativo de alimentação para a criança $(15,19,22,23)$.

Nos estudos em relação aos corticóides, foi avaliado o uso de Prednisona, que se enquadrou na categoria de risco B. Em gestantes, mulheres no período de amamentação ou em idade fértil, é necessário que os possíveis benefícios sejam avaliados em relação aos riscos potenciais para a mãe, para o feto ou recém nascido (15).

Na utilização de antifiséticos, o uso da Simeticona, com risco $C$, constatou-se que o risco para o feto é mínimo e o risco para a criança não pode ser estimado durante o período de lactação (15).

O Cetoconazol, um antifúngico, apresentou categoria de risco $\mathrm{C}$, atravessando a placenta, podendo provocar problemas fetais durante o primeiro trimestre da gravidez. Recomenda-se não prescrevê-lo durante a gravidez e a amamentação, dado que se excreta no leite materno e pode aumentar a possibilidade de kernicterus no lactante(6).

$\mathrm{Na}$ classe dos antiparasitários, o uso de Mebendazol, classe de risco $\mathrm{C}$, mostrou possíveis riscos associados à prescrição durante a gravidez, que devem ser pesados contra os benefícios terapêuticos esperados. É absorvido apenas em pequena quantidade e não há informações se é excretado no leite humano. Por esta razão, precauções devem ser tomadas quando mebendazol for administrado a lactantes(19).

Quando analisada a classe dos retinóicos, a Isotretinoina se enquadrou na categoria de risco D em relação a seu uso em grávidas e lactentes. $\mathrm{O}$ mais grave é a teratogenia, geração de defeitos permanentes no feto. Por isso, o uso de Isotretinoina é proibido em gestantes e é imprescindível que as mulheres realizem teste de gravidez antes de iniciar o tratamento. Em gestantes, o fármaco causa, além de inflamações de pele em vários graus, morte do feto ou malformações graves como hidrocefalia e microftalmia, em alguns casos associadas com defeitos nos vasos sanguíneos. Não é recomendado seu uso durante o período puerpério (24).

Por último, foi analisada a classe dos antidepressivos, avaliando os riscos da Amitriptilina, categoria de risco $\mathrm{C}$, em gestantes e lactantes e seus efeitos adversos no feto e no concepto. Este medicamento não deve ser utilizado por mulheres grávidas sem orientação médica. É detectável no leite materno e em razão do potencial para reações adversas graves causadas pela Amitriptilina em crianças, deve-se decidir entre descontinuar o medicamento ou a amamentação (25).

Devido a escassez de ações que envolvam informação aos profissionais de saúde e puérperas sobre o uso de medicamentos no período da gestação e amamentação, estudos que envolvem a identificação de medicamentos utilizados por gestantes e/ou puérperas, bem como o risco do uso desses medicamentos na gestação e ou lactação, torna-se clara a importância de estudos sobre esses fármacos e a divulgação de informações à respeito das mesmas para as nutrizes, e, consequentemente, qualificação dos profissionais da saúde quanto aos assuntos abordados neste trabalho $(3,25)$.

\section{CONCLUSÕES}

De acordo com os resultados encontrados a partir do estudo do uso dos medicamentos durante a gravidez/lactação, concluímos que o uso irracional pode ocasionar problemas irreversíveis ao feto. Devido à carência de informações na literatura, os prescritores devem redobrar o cuidado ao prescrever medicamentos a gestante e lactente, principalmente nos três primeiros meses de gestação, considerado um período crítico. Cabe a todos os profissionais de saúde informar as mulheres em idade fértil sobre o risco da utilização de medicamentos na gravidez, chamando atenção para o perigo potencial da automedicação, os efeitos adversos desses medicamentos tanto para elas quanto a criança.

O profissional farmacêutico é um importante elemento neste processo de prevenção, pois é o último profissional da saúde que tem contato com a paciente na dispensação. Dessa forma, espera-se uma mudança concreta nessa realidade, permitindo ao prescritor utilizar, de forma mais segura, os recursos terapêuticos disponíveis, e o farmacêutico desempenhar seu papel humanista na dispensação. 


\section{REFERÊNCIAS}

1. Castro CGSO, Paumgartten FJR, Silver LD. O uso de medicamentos na gravidez. Ciência \& Saúde Coletiva, 2004; 9(4):987-996.

2. Costa JM, Rocha LM, Cristiane MS, Abelha LL, Almeida KCA. Análise das prescrições medicamentosas em uma maternidade de belo horizonte e classificação de riscos na gestação e amamentação. Rev Bras Farm Hosp Serv Saúde. São Paulo 2012; v.3 n.1 32-36 jan./mar

3. Baldon JP, Correr CJ, Melchiors AC, Rossignoli P, Fernández-Llimós F, Pontarolo R. Conhecimento e atitudes de farmacêuticos comunitários na dispensação de medicamentos para gestantes. Pharm Practice 2006; 4(1): 38-43.

4. Brum LFS, Pereira P, Felicetti LL, Silveira RD. Utilização de medicamentos por gestantes usuárias do Sistema Único de Saúde no município de Santa Rosa (RS, Brasil). Ciência \& Saúde Coletiva, 2011; 16(5):2435-2442.

5. Silva, P. Farmacologia. 7ed. Rio de Janeiro: Guanabara Koogan, 2006. 1369 p. Il.

6. Brunton LL, Lazo JS, Parker K. Goodman e Gilman: as bases farmacológicas da terapêutica. 11.ed. Rio de Janeiro: McGraw-Hill Interamericana do Brasil, 2006.

7. Castro CGSO, Vera LEP, Luiza VL, Cosendey MAE, Freitas AM, Miranda FF, et al. Uso indicado e uso referido de medicamentos durante a gravidez. Cad. Saúde Pública, Rio de Janeiro, 2004; 20 Sup 1:S73-S82.

8. Fonseca MRCC, Fonseca E, Mendes GB. Prevalência do uso de medicamentos na gravidez: uma abordagem farmacoepidemiológica. Rev Saúde Pública 2002;36(2):205-12.

9. FDA: Food And Drug Administration. Highlights of Prescribing Information. 2010 [10 Mar. 2011] Disponível em: http://www. fda.gov.

10. Ballone GJ. Gravidez e Medicamentos. 2011 [10 Mar. 2011]; Disponível em: http://www.psiqweb. med.br.

11. Ciampo LAD, Ferraz IS, Daneluzzi JC, Ricco RG, Junior Carlos Eduardo Martinelli. Aleitamento materno e uso de medicamentos durante a lactação. Rev. paul. pediatr. [online]. 2007, vol.25, n.4, pp. 355-357.

12. Menguea SS, Schenkelb EP, Duncana BB, Schmidta MI. Uso de medicamentos por gestantes em seis cidades brasileiras. Rev Saúde Pública 2001;35(5):415-20.
13. Guerra GCB, Silva AQB, França LBS, Assunção PMC, Cabral RX, Ferreira A A. Utilização de medicamentos durante a gravidez na cidade de Natal, Rio Grande do Norte, Brasil. Rev Bras Ginecol Obstet. 2008; 30(1):12-8.

14. Boletín Terapêutico Atenolol - Bula. Laboratórios Bagó do Brasil. 2009 [5 Mar. 2011]; Disponível em: http://www.bago.com.br/ $\mathrm{web} / \mathrm{cgi} /$ cgilua.exe/sys/start.htm?sid=23\&lng=br.

15. Martis M.A. Bulário Eletrônco. 2009 [2 Mar. 2011] Disponível em: http://www.medicina net. com.br.

16. Brigss GG, Freeman RK, Yaffe SJ. Drugs in pregnancy and lactation: a reference guide to fetal and neonatal risk. 6 Ed. Philadelphia: Lippincott Williams \& Wilkins. 2002.

17. Fujisawa MO. Captopril - Bula. Medley Indústria Farmacêutica Ltda. 2011 [5 Mar. 2011] Disponível em: http://www.medley. com.br/portal/bula/captopril_comprimidos.pdf.

18. Marinho L. Ácido Fólico. 2009 [10 Mar. 2011] Disponível em: http://www.luismarinho.pt/ download s/1_indice_analises_2009_02_02.pdf

19. Ladeira JP. Bulário Eletrônco. 2009 [7 Mar. 2011] Disponível em: http://www.medicina net. com.br.

20. ANVISA: Agência Nacional de Vigilância Sanitária. Bulário Eletrônico da ANVISA. 2009 [14 Mar. 2011]; Disponível em: http:// www.pdamed.com.br/bulanv/pdamed_0001_00234_01600.php.

21. Ballone GJ. Gravidez e Psicotrópicos. Psiquiatria Geral. 2002 [5 Mar. 2011]; Disponível em: http://gballone.sites.uol.com.br/mulher/gravimed.htm. Acesso em: 5 de mar. 2011.

22. Luper L. Referencias Completas de medicamentos. 2010 [16 Mar. 2011] Disponível em: http://www.bulas. med.br.

23. Rombaldi AR, Galvão ALC, Grezzana GB. Anti-Hipertensivos e Diuréticos no Ciclo Gravídico-Puerperal. Revista da Sociedade de Cardiologia do Rio Grande do Sul. Ano XIV n 05 Mai/Jun/ Jul/Ago 2005.

24. Ferreira GN. Referências completas de Medicamentos. 2002 [12 Mar. 2011] Disponível em: http://www.bulas.med.br/p/roacutan+-3487.html.

25. Caria AT, Referências completas de Medicamentos. 2002 [16 Mar. 2011] Disponível em: http://www.bulas.med.br/bula/3753/ tryptanol. htm 